\title{
Role of Electrophysiological Studies in Diabetic Neuropathy
}

\author{
Vera Bril
}

\begin{abstract}
Electrophysiological studies are a reproducible and noninvasive method of assessing peripheral nerve function. The sensitivity of these methods has been validated in population-based and clinical studies, and the variability of test results is typically lower than with other noninvasive tests of nerve function. It has been recommended that standardized procedures including temperature control and equipment calibration be utilized with electrophysiological testing. The failure to detect significant changes in nerve conduction studies from clinical studies may be due to poor standardization of technique and inadequate sample size to detect differences. Baseline results from a multicenter, randomized trial indicate that different electrophysiological measures are highly correlated and reproducible. This and future studies that incorporate standardized techniques and an adequate sample size should be able to detect significant differences between treatments and identify effective therapeutic interventions for diabetic neuropathy.
\end{abstract}

\begin{abstract}
Résumé: Rôle des études électrophysiologiques dans la neuropathie diabétique. Les études électrophysiologiques sont une méthode reproductible noninvasive d'évaluation de la fonction du nerf périphérique. La sensibilité de ces méthodes a été validée par des études de population et des études cliniques et la variabilité des résultats est inférieure à celle autres épreuves d'évaluation de la fonction nerveuse. Il est recommandé que la marche à suivre standard, incluant le contrôle de la température et le calibrage le l'équipement, soit utilisée lors des épreuves électrophysiologiques. L'absence de changement significatif de la conduction nerveuse dans les études cliniques peut être due à une mauvaise standardisation de la technique et à un échantillon trop petit pour détecter une différence. Les valeurs de base obtenues au cours d'une étude multicentrique randomisée indiquent que différentes mesures électrophysiologiques sont hautement corrélées et reproductibles. Ces études ainsi que celles qui utiliseront des techniques standardisées et une taille d'échantillon adéquate devraient pouvoir détecter des différences significatives entre les traitements et identifier quelles sont les interventions thérapeutiques qui sont efficaces dans la neuropathie diabétique.
\end{abstract}

Can. J. Neurol. Sci. 1994; 21: Suppl. 4-S8-S12

Diabetic neuropathy is a common complication of diabetes that eventually affects the majority of diabetic patients and is associated with significant morbidity and disability. A survey of studies from the literature shows that the percentage of patients with diagnosed neuropathy varies depending on the measure used for assessment.' Historically, when the diagnosis was based on nerve conduction velocity determinations, up to $100 \%$ of the patients have abnormalities. ${ }^{2}$ Improved techniques and knowledge of electrodiagnostic procedures have helped establish its role in the assessment of neuropathy and its use in clinical trials to evaluate the response to therapeutic interventions are reviewed here.

\section{Electrophysiological ASSESSMENT}

Electrophysiological testing is a valuable component of the overall evaluation of peripheral symmetrical polyneuropathies. Electrophysiological studies are more sensitive than clinical examinations and the least variable noninvasive measure of neuropathy. Up to $75 \%$ of asymptomatic patients with a normal or abnormal clinical examination may have nerve conduction abnormalities that are typical of neuropathy. ${ }^{3-5}$ Approximately
$10 \%$ of unselected patients screened in a diabetes clinical will have symptoms of neuropathy, another $10 \%$ will have abnormalities on the neurological examination, and an additional $40 \%$ will have abnormal nerve conduction studies.,

In 1988, a conference was held in San Antonio to develop standardized terminology and assessments for diabetic neuropathy. ${ }^{7}$ Five different measures were declared: symptoms, clinical findings, nerve conduction studies, quantitative sensory testing, and quantitative autonomic testing. At the conclusion of the conference it was recommended that an abnormality in two or more of these measures was needed in order to diagnose peripheral neuropathy.

The role of nerve conduction studies in neuropathy can be evaluated with population studies in which diabetic patients with neuropathy are compared with a normal population to determine

From the Division of Neurology, The Toronto Hospital, University of Toronto, Toronto. Reprint requests to: Vera Bril, MD, FRCPC, Division of Neurology, ENI 1-209. Toronto Hospital, 200 Elizabeth Street, Toronto, Ontario, Canada M5G 2C4 
patterns of abnormality. Typically, lower limbs are affected more and earlier than upper limbs. The conduction velocities are mildly slowed in diabetic patients, although not to the degree that is seen in Guillain-Barre syndrome. Motor and sensoryevoked amplitude responses also are reduced. The sensitivity of nerve conduction studies has been well validated in numerous clinical studies, and it is the least variable measure of neuropathy, if standardized techniques are used which define testing procedures and equipment specifications. ${ }^{8}$

The Rochester Diabetic Neuropathy Study is an ongoing prospective, longitudinal study of a cohort of diabetic patients in Rochester, Minnesota. ${ }^{9}$ As part of that study, the validity of the San Antonio criteria was evaluated. The most interesting finding was that nerve conduction studies were the best measure of neuropathy. ${ }^{10}$ In the Rochester study, patients underwent a standardized assessment that included the Neuropathy Symptom Score, the Neuropathy Disability Score, nerve conduction studies, quantitative sensory testing, and quantitative autonomic testing. The criteria for neuropathy consisted of the finding of an abnormality in at least two of these assessments with at least one of the abnormalities being nerve conduction or quantitative autonomic testing. These criteria were evaluated to see which assessment was the most sensitive and specific for neuropathy. There was a general association between the different assessment methods. Nerve conduction studies and autonomic testing were the most sensitive and objective measures for detecting neuropathy. However, the Neuropathy Symptom Score, Neuropathy Disability Score, quantitative sensory testing, and nerve conduction studies were optimal for determining the severity of neuropathy. The strongest correlations were between the disability score, nerve conduction studies, and quantitative autonomic testing.

\section{VARIABILITY OF Electrophysiological TESTING}

The variability of a diagnostic test is important, especially when it is used to monitor the course of a disease. For example, in a double-blind, placebo-controlled trial of the aldose reductase inhibitor (ARI) ponalrestat in 259 patients with diabetic neuropathy, no significant differences were observed between treatment groups after 18 months." One explanation for the failure to detect an effect with active drug treatment was the high coefficients of variation for the measures of efficacy used in this study (Table 1). Consequently, the study had very little power to

Table 1. Coefficients of variation for different measures of efficacy from a study of ponalrestat.

\begin{tabular}{lc}
\hline Variable & Coefficient of Variation (\%) \\
\hline Vibration threshold (great toe) & $8.7-29.7$ \\
Conduction velocity & \\
$\quad$ Median sensory & $1.6-12.7$ \\
Median motor & $2.9-9.5$ \\
Sural & $0.9-20.9$ \\
Peroneal & $0.7-11.5$ \\
Amplitude & \\
Sural & $8.9-42.8$ \\
Peroneal & $13.4-80.4$ \\
\hline
\end{tabular}

(Sundvist et al. ${ }^{11}$, with permission) detect a significant difference between a potentially active drug and a placebo.

The coefficients of variation for different objective measures of efficacy used in a long-term study of tolrestat for diabetic neuropathy are shown in Table $2 .^{12}$ The lowest variability in these studies was in motor conduction velocity, which varied from $4 \%$ to $5 \%$. Data from our laboratory show a variation of $3.7 \%$ to $3.8 \%$ for motor conduction velocity, $4.1 \%$ to $6.2 \%$ for sensory conduction velocity, $28 \%$ for sural nerve amplitude, and $16 \%$ for peroneal amplitude.

Few studies reported in the literature provide an adequate number of patients to detect a significant difference between two or more interventions. The importance of adequate sample size is demonstrated by an examination of the relationship between the duration of diabetes mellitus and sural nerve amplitude. With a small number of patients, the sural amplitude appears to increase with the duration of diabetes, but with a larger sample size, the amplitude decreases with the duration of diabetes (personal observation). The coefficient of variation for an assessment method can be used to perform a power calculation to determine the minimum number of patients needed per treatment group to show significant changes. For instance, to obtain a two-sided alpha of 0.05 and a power of 0.9 with variability of measures such as shown in Table 2, 42 patients per group are needed to detect a $2.5 \mathrm{~m} / \mathrm{sec}$ change in nerve conduction velocity, 175 per group are needed to detect significant amplitude changes, and more than 550 per group are needed to detect a significant change in vibration perception threshold. ${ }^{8}$

Although nerve conduction studies must be performed with meticulous technique, they are readily available, reliable, and reproducible when standardized methods are used. Nevertheless, it is difficult to compare results of nerve conduction studies between the various published reports because of the failure to employ standardized testing procedures across studies. The problem was addressed in 1992 at a joint American Diabetes Association and American Academy of Neurology consensus meeting. ${ }^{13}$ It was recommended that standardized protocols for electrophysiological testing should include temperature control, calibration of the equipment, averaging for sensory responses, a definition of the recording and stimulating sites, and specific measurement of sensory nerve and compound muscle action potentials.

Table 2. Coefficients of variation (\%) for objective measures of efficacy from a trial of tolrestat for diabetic neuropathy.

\section{Variable} Coefficient of Variation (\%)

Quantitative sensory testing

Vibration threshold

$14.8-22.2$

Nerve amplitude

Sensory

28.3-35.6

Motor

$19.6-32.8$

Nerve conduction velocity

Sensory

5.4-9.1

Motor

$3.9-4.7$

(Santiago et al. ${ }^{12}$, with permission) 


\section{Relationship Between Electrophysiology AND MORPHOMETRY}

Nerve conduction velocity is determined by the rate at which the action potential is generated. The rate is variable and depends on current flow along the axon, the depolarization threshold of the membrane, and temperature. In myelinated fibers, action potentials are generated only at the nodes of Ranvier. Axoplasmic resistance inhibits flow, whereas myelin capacitance and conductance decrease with myelin thickness, which results in more rapid conduction. Thus, for a given axon diameter, conduction velocity increases with myelin thickness. In damaged nerves, when Wallerian degeneration or axonal atrophy and loss are present, regeneration, repair, and Schwann cell proliferation occur. More nodes and shorter internodal lengths replace the normal structure of the fiber, resulting in shorter internodal distances and slower conduction velocities. With nerve remyelination, myelin is thinner relative to axon diameter, and conduction velocity, even in repaired fibers, remains slow and abnormal. Complete normalization of conduction velocity will not occur despite adequate nerve regeneration.

Although nerve conduction studies are the least variable noninvasive method for assessing diabetic neuropathy, nerve histomorphometry is the optimal research method for assessing neuropathic changes in the diabetic nerve. The reproducibility and sensitivity of nerve morphometry for assessing diabetic neuropathy were determined from sural nerve biopsies obtained post mortem from both diabetic and nondiabetic subjects. ${ }^{14}$ The results showed that several morphometric measures, including fiber density, fiber size, and myelin wrinkling, are reliable, reproducible, and sensitive measures of nerve fiber loss and atrophy and exhibit low variability. A previous study by Sima and associates showed a correlation between nerve morphometry and nerve conduction velocity in patients with diabetic neuropathy. ${ }^{15}$ Thus, because morphometric analysis is highly reproducible and sensitive, it can be used to establish the relevance of nerve conduction studies in diabetic neuropathy.

It has been hypothesized that if sural nerve morphology represents diffuse neuropathy, then sural nerve pathology should correlate with changes in other nerves. Therefore, median sensory nerve amplitude and conduction velocity as well as median motor nerve amplitude and conduction velocity should reflect sural nerve fiber density. As the nerve degenerates, structural alterations should result in reduction in nerve amplitude and a secondary slowing of conduction velocity in multiple nerves. The relationship between nerve structure and function is being evaluated in a multicenter North American study, in which over 400 patients with diabetic neuropathy were randomly assigned to tolrestat or placebo. Whole sural nerve biopsy was performed at baseline and will be repeated after 72 weeks of treatment to assess the effects of treatment on nerve morphometry and functional end points, including nerve conduction studies. Preliminary review of the baseline data shows correlations between nerve conduction measures and morphometry.

Data from clinical trials with an ARI or an intensified insulin regimen show that treatment stabilizes nerve conduction velocity deterioration. ${ }^{16-20}$ In one trial, deterioration of nerve conduction velocity decreased in patients in the placebo group, and nerve conduction velocity stabilized in the group treated with an aldose reductase inhibitor. ${ }^{15}$ The Stockholm Diabetes
Intervention Study followed 96 insulin-dependent diabetic patients, who were randomly assigned to regular or intensified insulin regimens and followed for 7.5 years. ${ }^{19}$ Patients on the regular insulin regimen experienced a significant $(P<.05)$ decrease in conduction velocity of the sural, peroneal, and tibial nerves compared with those in the intensified therapy group.

Figure 1 depicts the expected change in conduction velocity over time in normal controls, in untreated diabetic patients with neuropathy, and in diabetic patients with neuropathy who were treated with an ARI. ${ }^{21-23}$ Because of the extensive structural nerve damage, it is unrealistic to expect that treatment in the neuropathy patient will improve nerve conduction velocity to the level of a nondiabetic control. A more realistic expectation is that the rate of decline in nerve conduction velocity will slow to approach that of the nondiabetic patient.

\section{Impact of Treatment on Nerve Conduction}

Results from the Diabetes Control and Complications Trial provide some insight into the potential benefits of intensive insulin therapy in the treatment of diabetic complications. ${ }^{20}$ Consistent with other studies of improved glycemic control, neither the conventional nor the intensive therapy group attained a normal glycated hemoglobin, and only $44 \%$ of the intensively treated group achieved the target level of $<6.05 \%$ at least once. Only $5 \%$ of patients maintained an average value in that range. Nevertheless, with intensive therapy, the risk of clinical neuropathy was reduced by $61 \%$. During the study, however, new abnormalities in nerve conduction studies were detected in $19 \%$ of patients in the intensive therapy group, which suggests that elevated blood glucose has a potent adverse effect on the nerve. A 3.3-fold increase in the incidence of severe hypoglycemic reactions, which included coma, seizures, emergency room admission, and any episode that required medical assistance, was observed in the intensive treatment group. Thus, a beneficial reduction in the risk of complications was apparent, but the costs of intensive therapy are less clear.

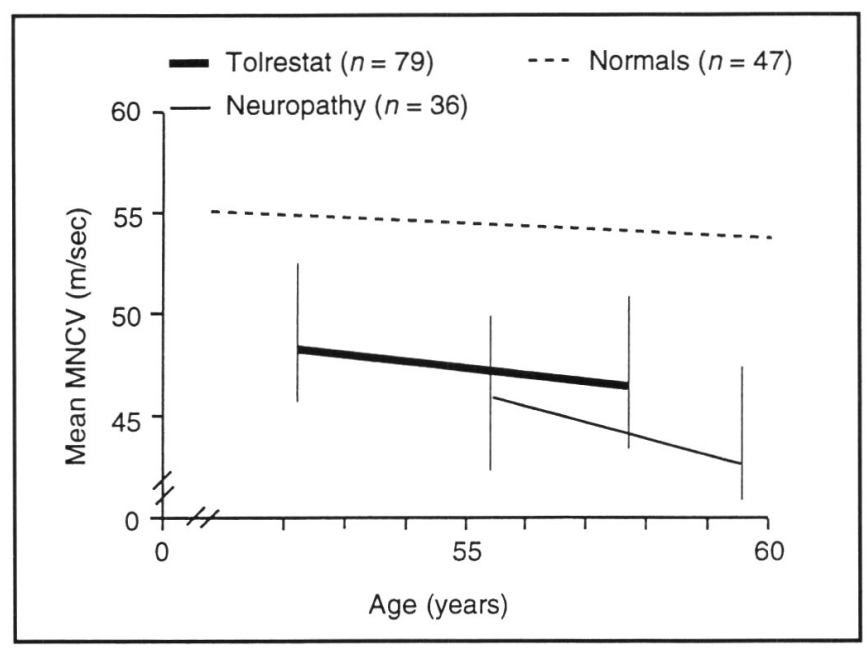

Figure 1: Change in median motor nerve conduction velocity in nondiabetic patients, diabetic patients with neuropathy, and neuropathy patients treated with tolrestat (Boulton et al. ${ }^{21}$, with permission; Pfeifer et al. ${ }^{23}$, with permission). 
A number of clinical trials have been performed with ARIs, in which nerve conduction velocity was measured. ${ }^{11.17 .24}$ In general, treatment slowed the rate of decline, although conduction velocity was not normalized. In one long-term trial, the effects of tolrestat were studied in patients with advanced neuropathy and who had received open-label tolrestat for more than four years. ${ }^{12}$ Patients were given the option of continuing tolrestat or switching to placebo in a double-blind manner. They also were allowed to switch back to the alternative treatment within three months. Those who continued on tolrestat showed continued improvement in nerve conduction velocity, whereas conduction velocity deteriorated in patients who switched to placebo (Figure 2).

Baseline data from the clinical site in Toronto for the North American study of tolrestat provide examples of the information that can be obtained from nerve conduction studies (Table 3 ).

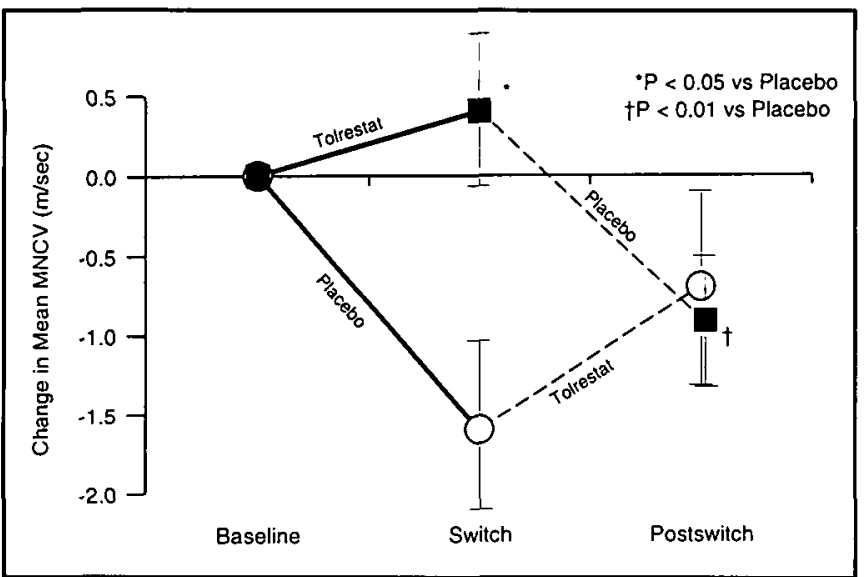

Figure 2: Change in mean motor nerve conduction velocity of four nerves before and after elected switch between tolrestat and placebo (Santiago et al. ${ }^{\prime 2}$, with permission).

Table 3. Baseline data from 100 patients enrolled in a North American multicenter study of tolrestat.

\begin{tabular}{lc}
\hline Variable & Mean \pm SD* \\
Age (years) & $52.8 \pm 10.6$ \\
Duration of diabetes (years) & $12.6 \pm 9.0$ \\
Type 1:type $2(\%)$ & $25: 75$ \\
Glycated hemoglobin & $10.3 \pm 2.3$ \\
Male:female & $73: 27$ \\
Sensory score (maximum 64$)$ & 19.1 \\
Median motor amplitude $(\mathrm{mV})$ & $6.6 \pm 2.4$ \\
Median motor conduction velocity $(\mathrm{m} / \mathrm{s})$ & $49.1 \pm 4.8$ \\
Median sensory amplitude $(\mu \mathrm{V})$ & $18.2 \pm 9.2$ \\
Median sensory conduction velocity $(\mathrm{m} / \mathrm{s})$ & $52.1 \pm 4.7$ \\
Peroneal amplitude $(\mathrm{mV})$ & $3.3 \pm 2.0$ \\
Peroneal conduction velocity $(\mathrm{m} / \mathrm{s})$ & $38.4 \pm 5.0$ \\
Right sural amplitude $(\mu \mathrm{V})$ & $4.8 \pm 3.3$ \\
Right sural conduction velocity $(\mathrm{m} / \mathrm{s})$ & $37.2 \pm 5.7$ \\
Left sural amplitude $(\mu \mathrm{V})$ & $4.8 \pm 3.0$ \\
Left sural conduction velocity $(\mathrm{m} / \mathrm{s})$ & $36.6 \pm 5.5$
\end{tabular}

* Mean \pm standard deviation
Patients with mild neuropathy and a mean sensory score of 19 based on a maximum sensory score of 64 were recruited. Sensory scores were a numerical summation from an assessment of pin prick, light touch, vibration, and position performed bilaterally on the index finger and first toe. In the first 100 patients, the average duration of diabetes was 12 years, and $25 \%$ of patients had type I diabetes. Mean values for nerve conduction velocity and amplitude are shown in Table 3. No significant differences were observed between patients with type I and type II diabetes for sensory scores or electrophysiologic measures (Figure 3).

Correlation matrices were constructed to compare the standard fixed-distance method and an alternative variable-distance method for assessment of sural responses. High correlations were observed between the standard and alternative methods for both sural nerve conduction velocity and amplitude (Table 4). The coefficient of variation was slightly greater for fixed-distance amplitudes compared to variable-distance amplitudes ( $26.9 \%$ and $28.1 \%$ compared to $26.6 \%$ and $24.7 \%$, respectively for right and left sural nerves). In addition, good correlations were observed for conduction velocity and amplitude of nerves obtained from the left and right extremity (Figure 4). Median sensory, sural, and peroneal motor conduction velocity tended to decrease with increasing glycated hemoglobin. The magnitude of change was not greater because patients with minimal neuropathy, presence of sural and peroneal responses, and without

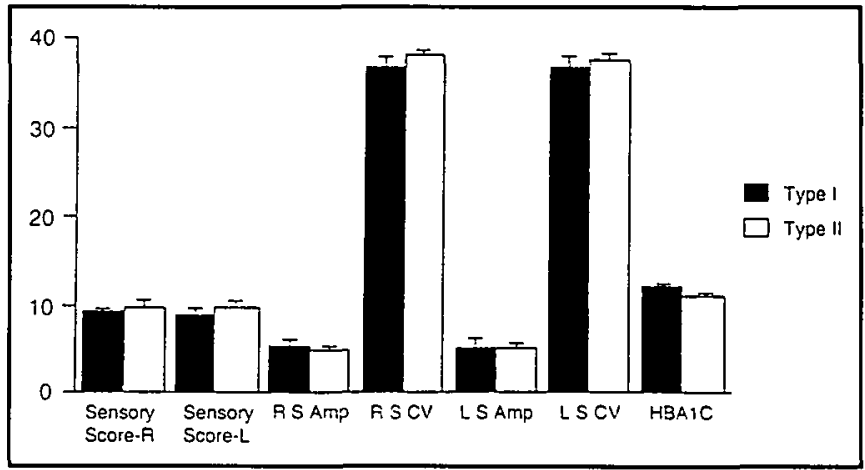

Figure 3: Comparison of baseline sensory scores and electrophysiological parameters in type I and type $1 /$ patients in 100 patients from a North American trial of tolrestat (values are mean \pm standard ermor).

Table 4. Correlations between sural nerve amplitude and conduction velocity obtained by two different electrophysiologic methods at baseline in 100 patients from a North American trial of tolrestat.

\begin{tabular}{llll}
\hline Standard Method & Alternative Method & $\mathbf{r}$ & $\mathbf{r}^{\mathbf{2}}$ \\
Amplitude & & \\
$\quad$ Maximum right sural & Right sural & 0.948 & 0.898 \\
$\quad$ Maximum left sural & Left sural & 0.943 & 0.889 \\
$\quad$ Right sural & Left sural & 0.844 & 0.713 \\
Conduction velocity & & & \\
$\quad$ Maximum right sural & Right sural & 0.949 & 0.901 \\
Maximum left sural & Left sural & 0.945 & 0.893 \\
Right sural & Left sural & 0.871 & 0.759
\end{tabular}




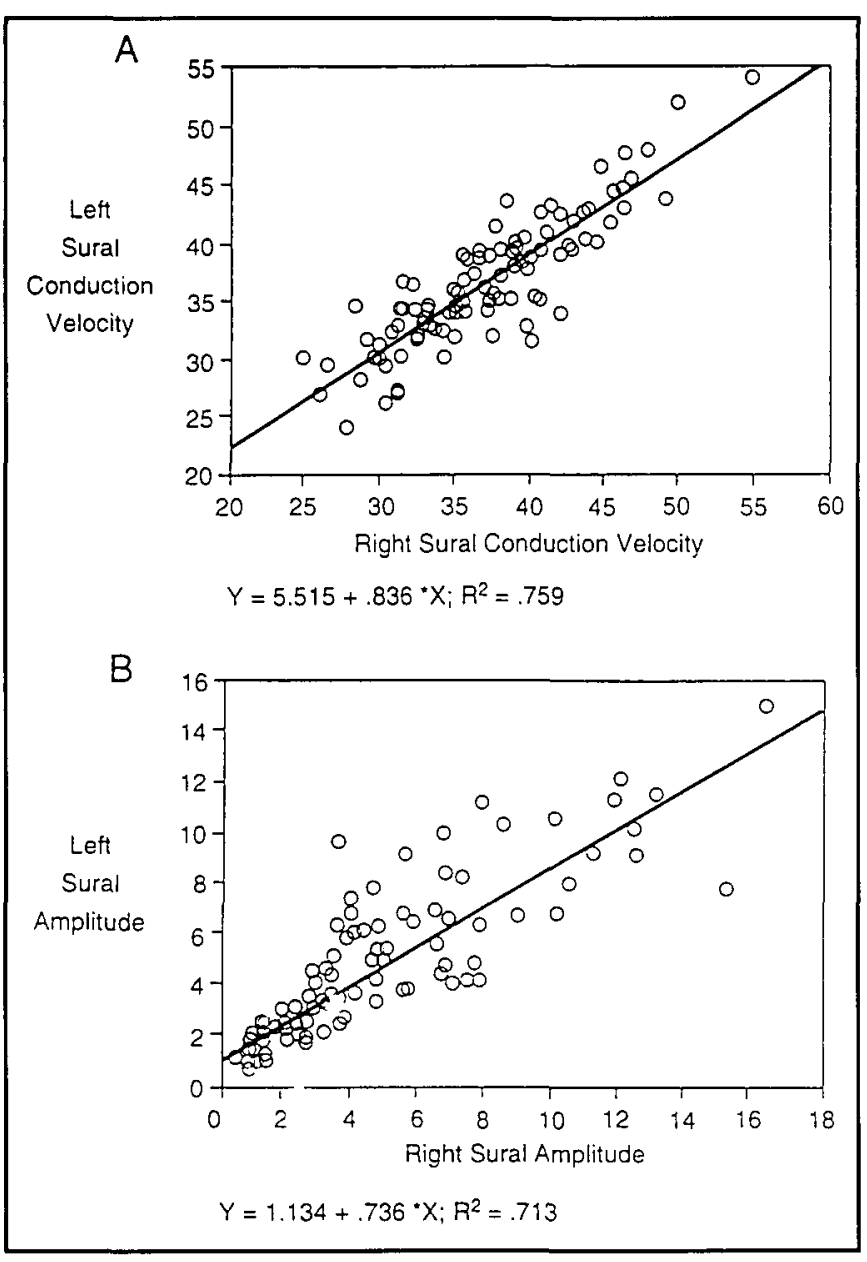

Figure 4: Correlation between left and right sural nerve conduction velocity (panel $A$ ) and left and right sural nerve amplitude (panel $B$ ) at baseline in 100 patients from a North American trial of tolrestat.

ulceration were selected for this trial. In addition, patients were required to have an abnormality in two of four San Antonio criteria: symptoms, conduction velocity, autonomic testing, or quantitative sensory testing.

\section{SUMMARY}

Electrophysiological testing is sensitive, noninvasive, widely available, and the least variable measure of diabetic neuropathy. It provides a quantitative assessment that can be followed longitudinally. However, a study must have adequate power and duration of follow-up to detect a change. Nerve conduction amplitude is used less commonly in clinical trials because of large variability, but future studies may have adequate power to detect amplitude changes.

The goals of early intervention in neuropathy are to prevent progression to end-stage nerve degeneration. Small changes in conduction velocity are due to improved function at the node of Ranvier and are the expected benefits of therapeutic interventions in diabetic neuropathy. Interpretation of clinical trials of neuropathy requires realistic functional expectations of the benefits of treatment.

\section{REFERENCES}

1. Vinik Al, Holland MT, Le Beau JM, et al. Diabetic neuropathies. Diabetes Care 1992; 15: 1926-1975.

2. Diabetes Data. U.S. Department of Health, Education, and Welfare National Institutes of Health. DHEW Publication No. (NIH) 78 1468. Washington, DC: U.S. Government Printing Office, 1978.

3. Brown MR, Dyck PJ, McClearn GE, et al. Central and peripheral nervous system complications. Diabetes 1982; 31 (Suppl 1, part 2): $65-70$.

4. Lamontagne A, Buchthal F. Electrophysiological studies in diabetic neuropathy. J Neurol Neurosurg Psychiatry 1970; 33: 442-452.

5. Young RJ, Ewing DJ, Clarke BF. Nerve function and metabolic control in teenage diabetics. Diabetes 1983; 32: 142-147.

6. Boulton AJ, Knight G, Drury J, Ward JD. The prevalence of symptomatic, diabetic neuropathy in an insulin-treated population. Diabetes Care 1985; 8: 125-128.

7. Consensus Statement of the American Diabetes Association and the American Academy of Neurology: Report and Recommendations of the San Antonio Conference on Diabetic Neuropathy. Diabetes 1988; 37: 1000-1004.

8. Macleod AF, Till S, Sonksen PH. Discussion of the clinical trials of the aldose reductase inhibitor tolrestat. Int Proc J 1991; 4: 17-24.

9. Dyck PJ, Kratz KM, Lehman KA, et al. The Rochester Diabetic Neuropathy Study: design, criteria for types of neuropathy, selection bias, and reproducibility of neuropathic tests. Neurology 1991; 41: 799-807.

10. Dyck PJ, Karnes JL, O'Brien PC, et al. The Rochester Diabetic Neuropathy Study: reassessment of tests and criteria for diagnosis and staged severity. Neurology 1992; 42: 1164-1170.

11. Sundkvist G, Armstrong F, Bradbury J, et al. for the United Kingdom/Scandinavian Ponalrestat Trial. Peripheral and autonomic nerve function in 259 diabetic patients with peripheral neuropathy treated with ponalrestat (an aldose reductase inhibitor) or placebo for 18 months. J Diab Complications 1992; 6: $123-130$.

12. Santiago JV, Sonksen PH, Boulton AJM, et al. Withdrawal of the ARI, tolrestat in patients with diabetic neuropathy: effect on nerve function. J Diab Complications 1993; 7: 170-179

13. Consensus Statement. Proceedings of a Consensus Development Conference on Standardized Measures in Diabetic Neuropathy. Neurology 1992; 42: 1823-1829.

14. Sima AAF, Brown MR. Prashar A, et al. The reproducibility and sensitivity of sural nerve morphometry in the assessment of diabetic peripheral polyneuropathy. Diabetologia 1992; 35: 560-569.

15. Sima AAF, Bril V, Nathaniel V, et al. Regeneration and repair of myelinated fibers in sural-nerve biopsy specimens from patients with diabetic neuropathy treated with sorbinil. N Engl J Med 1988; 319: 548-555.

16. Agardh CD, Rosen I, Schersten B. Improvement of peripheral nerve function after institution of insulin treatment in diabetes mellitus. A case-control study. Acta Med Scand 1983; 213: 283-287.

17. Boulton AJM, Levin S, Comstock JA. A multicentre trial of the aldose-reductase inhibitor, tolrestat, in patients with symptomatic diabetic neuropathy. Diabetologia 1990; 33: 431-437.

18. Judzewitsch RG, Jaspan JB, Polonsky KS, et al. Aldose reductase inhibition improves nerve conduction velocity in diabetic patients. N Engl J Med 1983; 308: 119-125.

19. Reichard P, Nilsson B-Y, Rosenquist U. The effect of long-term intensified insulin treatment on the development of microvascular complications of diabetes mellitus. N Engl J Med 1993; 329: 304-309.

20. Diabetes Control and Complications Trial Research Group. The effect of intensive treatment of diabetes on the development and progression of long-term complications in insulin-dependent diabetes mellitus. N Engl J Med 1993; 329: 977-986.

21. Boulton AJM, Armstrong WD, Scarpello JHB, Ward JD. The natural history of painful diabetic neuropathy. Postgrad Med J 1983; 59: 556-559.

22. Dorfman LJ, Bosley TM. Age-related changes in peripheral and central nerve conduction in man. Neurology $1979 ; 29: 38-44$.

23. Pfeifer MA, Weinberg CR, Cook DL, et al. Correlations among autonomic, sensory, and motor neural function tests in untreated non-insulin dependent diabetic individuals. Diabetes Care 1985; 8: 576-584.

24. Macleod AF, Boulton AJM, Owens DR, et al. A multicentre trial of the aldose-reductase inhibitor tolrestat, in patients with symptomatic diabetic peripheral neuropathy. Diabetes Metab 1992; 18: 14-20. 